\title{
Switching to anastrozole plus goserelin vs continued tamoxifen for adjuvant therapy of premenopausal early-stage breast cancer: preliminary results from a randomized trial
}

This article was published in the following Dove Press journal:

Cancer Management and Research

Jian-wei Li, ${ }^{1,2, *}$ Guang-yu Liu, ${ }^{1,2, *}$ Ya-jie Ji, ${ }^{3, *}$ Xia Yan, ${ }^{1,2}$ Da Pang, ${ }^{4}$ Ze-fei Jiang, ${ }^{5}$ De-dian Chen, ${ }^{6}$ Bin Zhang, ${ }^{7}$ Bing-he $\mathrm{Xu},{ }^{8} \mathrm{Z}$ hi-ming Shao ${ }^{1,2}$

'Department of Breast Surgery, Fudan University Shanghai Cancer Center, Shanghai, People's Republic of China; ${ }^{2}$ Department of Oncology, Shanghai Medical College, Fudan University, Shanghai, People's Republic of China; ${ }^{3}$ Department of Breast Surgery, Yueyang Hospital of Integrated Traditional Chinese and Western Medicine, Shanghai University of Traditional Chinese Medicine, Shanghai, People's Republic of China; ${ }^{4}$ Department of Breast Surgery, The Third Affiliated Hospital of Harbin Medical University, Harbin, People's Republic of China; ${ }^{5}$ Department of Oncology, 307 Hospital of People's Liberation Army, Beijing, People's Republic of China; ${ }^{6}$ Department of Breast Diseases, Cancer Hospital of Yunnan Province, Kunming, People's Republic of China; ${ }^{7}$ Department of Breast Surgery, Cancer Hospital of Liaoning Province, Shenyang, People's Republic of China; ${ }^{8}$ Department of Oncology, Cancer Hospital of Chinese Academy of Medical Sciences, Beijing, People's Republic of China

*These authors contributed equally to this work

Correspondence: Zhi-ming Shao Department of Breast Surgery, Shanghai Cancer Center, Fudan University, 399 Ling-Ling Road, Shanghai 200032, People's

Republic of China

$\mathrm{Tel}+862164175590$

Fax +86 2I 64434556

Email zhimingshao@fudan.edu.cn
Purpose: To assess the efficacy, safety, and quality-of-life impact of switching adjuvant treatment in hormone receptor-positive primary breast cancer patients who are still premenopausal after 2-3 years of tamoxifen therapy to anastrozole plus goserelin as compared with continuing tamoxifen over a total period of 5 years.

Patients and methods: Hormone receptor-positive, premenopausal, lymph node-positive, or tumor size $\geq 4 \mathrm{~cm}$ breast cancer patients who had received tamoxifen for 2-3 years were randomly assigned to continue tamoxifen treatment (TAM group) or switch to adjuvant anastrozole plus goserelin (ADD group) and continue treatment for another 2-3 years (total treatment duration 5 years). Endpoints evaluated were adverse events (AEs), changes in bone mineral density, quality of life, and disease-free survival-related events.

Results: A total of 62 patients (33 in the ADD group and 29 in the TAM group) were evaluated. Grade 3-4 drug-related AEs occurred in five patients (15.2\%) in the ADD group vs none in the TAM group. In the ADD group, arthralgias were the most common AEs ( $5 / 33$ patients; $15.2 \%$ ), and three patients in this group were discontinued because of AEs. Treatment was temporarily suspended due to AEs in three patients $(9.1 \%)$ in the ADD group and one patient $(3.4 \%)$ in the TAM group. Compared with continuing TAM therapy, switching to anastrozole plus goserelin did not result in any worsening of bone mineral density or quality of life. During a median follow-up of 34 months, five patients (15.2\%) in the ADD group had disease-free survival events vs four patients $(13.8 \%)$ in the TAM group.

Conclusion: For early-stage breast cancer patients who remain premenopausal following 2-3 years of adjuvant tamoxifen therapy, switching to anastrozole plus goserelin therapy was safe with tolerable adverse effects. However, it did not show superior efficacy compared to remaining on tamoxifen treatment.

Trial Registration: ClinicalTrials.gov (identifier NCT01352091).

Keywords: breast cancer, adjuvant therapy, anastrozole, tamoxifen, GnRH analogs, aromatase inhibitors

\section{Introduction}

Breast cancer is the most common type of cancer among women and about $75 \%$ of these cancers are hormone dependent. ${ }^{1}$ In People's Republic of China, around 56\% of breast cancer patients are premenopausal, of whom about $60 \%$ have estrogen receptor (ER)-positive cancer. ${ }^{2}$ In these patients, endocrine therapy is necessary, the major categories of which are selective ER modulators, third-generation aromatase 
inhibitors (AIs), ovarian suppressive therapies, and selective ER degraders. AI is the standard treatment for ER-positive postmenopausal early-stage breast cancer. Patients who are in postmenopausal stage during the use of adjuvant tamoxifen therapy can benefit from switching to an $\mathrm{AI} ;{ }^{3,4}$ for premenopausal patients, tamoxifen remains the standard adjuvant endocrine therapy. ${ }^{5}$

Gonadotropin-releasing hormone analog $(\mathrm{GnRH}-\mathrm{a})$ is often used as a method of drug-induced ovarian ablation. Following standard adjuvant therapy (chemotherapy and radiotherapy) in premenopausal patients with early-stage breast cancer, treatment with a GnRH-a can significantly improve event-free survival and the overall survival rate. ${ }^{6-8}$ Clinical studies have shown that in locally advanced premenopausal breast cancer, an AI combined with a GnRH-a may have better efficacy and tolerability. ${ }^{9-11}$ As AI+GnRH-a therapy can lower estrogen levels in vivo, ${ }^{12}$ for patients with early-stage breast cancer who are still premenopausal, it is not known whether it is better to switch ongoing tamoxifen treatment to an AI plus a GnRH-a, and this remains to be determined in clinical studies.

The primary objective of the present study was to assess the safety and quality-of-life impact of switching adjuvant treatment of hormone receptor-positive primary breast cancer patients who are still premenopausal after 2-3 years of tamoxifen therapy to $\mathrm{AI}+\mathrm{GnRH}$-a therapy (anastrozole + goserelin) as compared with continuing tamoxifen over a total period of 5 years. Although the present study was originally designed to enroll 670 patients, soon after enrollment had commenced, the results of several large clinical trials regarding adjuvant endocrine therapy were published. These include the ABCSG12 trial, which showed that compared with combined GnRH-a and tamoxifen therapy, combined GnRH-a and AI therapy was not superior in either efficacy nor safety. ${ }^{13}$ Two other studies, Tamoxifen and Exemestane Trial (TEXT) and Suppression of Ovarian Function Trial (SOFT), have reported their combined analysis data that 5 years of adjuvant treatment with exemestane plus ovarian suppression significantly reduced recurrence compared to tamoxifen plus ovarian suppression. ${ }^{14}$ Consequently, we decided to suspend enrollment of patients in our study after 62 patients had been randomized and followed up, and the present analysis is a summary of the safety, quality of life, and preliminary efficacy data of this population.

\section{Patients and methods Study design}

We conducted a randomized, open-label, multicenter clinical trial in hormone receptor-positive, primary breast cancer patients with early-stage, high-risk disease who were still premenopausal after standard surgery, chemotherapy, radiotherapy, and 2-3 years of tamoxifen (or toremifene) therapy. After the patients signed the informed consent, they were randomly allocated to one of two treatment groups stratified according to the number of positive lymph nodes $(0,1-3,4+)$, receipt of neoadjuvant chemotherapy, and tumor size ( $\geq 4$ or $<4 \mathrm{~cm}$ ). Patients received either 5 years' tamoxifen therapy (TAM/control group) or 2-3 years of tamoxifen followed by a switch to 2-3 years' anastrozole plus goserelin therapy (ADD group). Thus, all patients received adjuvant endocrine therapy for a total of 5 years.

The study was approved by the Fudan University Cancer Hospital Ethic Committee for Clinical Investigation (approval number: 080258-8-0904A). The study was carried out in accordance with the Declaration of Helsinki. Written informed consent was obtained from all patients prior to enrollment. It was registered with ClinicalTrials.gov (identifier NCT01352091).

\section{Eligibility criteria}

Eligible patients were women with histologically confirmed hormone receptor-positive (ER-positive and/or progesterone receptor-positive) primary breast cancer with ipsilateral axillary or internal mammary lymph node-positive disease or tumor size $\geq 4 \mathrm{~cm}$. All patients were required to have undergone standard treatment of primary breast cancer (ie, surgery, chemotherapy, radiotherapy), and those who had received neoadjuvant chemotherapy were required to have been compliant with such treatment. Endocrine therapy with tamoxifen (or toremifene) was started 6 weeks after standard treatment. Randomization to the study groups was performed after at least 2 (but no more than 3) years' adjuvant tamoxifen (or toremifene) therapy, provided patients showed no evidence of metastasis or recurrence and were still premenopausal 4 weeks before they were randomized. "Premenopausal" was defined as normal menstruation; amenorrhea of not more than 6 months' duration prior to enrollment and circulating blood levels of estradiol (E2), follicle-stimulating hormone $(\mathrm{FSH})$ and luteinizing hormone $(\mathrm{LH})$ that did not reach postmenopausal levels; age $<40$ years with amenorrhea of not more than 1 year' duration and circulating blood levels of E2, FSH, and LH that did not reach postmenopausal levels; or hysterectomy prior to enrollment with one or both ovaries retained and circulating blood levels of E2, FSH, and LH that did not reach postmenopausal levels. Patients were also required to have a negative pregnancy test and employ effective contraceptive measures during the study. 
The main exclusion criteria were the presence of a metastatic tumor, metachronous bilateral breast cancer, other malignant tumors in the past 5 years (except for cervical carcinoma in situ or cured basal cell or squamous cell carcinomas), other nonmalignant systemic diseases that could affect patients' long-term follow-up, previous ovariectomy, and HER-2 overexpression with ongoing use or planned use of adjuvant trastuzumab therapy.

\section{Treatments}

Following randomization, patients in the ADD group were given goserelin $\left(\right.$ Zoladex $\left.^{\circledR}\right) 3.6 \mathrm{mg}$ by subcutaneous injection every 28 days ( \pm 2 days) commencing at 30 days after enrollment and oral anastrozole (Arimidex ${ }^{\circledR}$ ) $1 \mathrm{mg}$ daily beginning 1 day after the first injection of goserelin. Patients in the TAM (control) group continued to take their originally prescribed tamoxifen dosages. All other anticancer treatments, including chemotherapy regimens, immunotherapy/ biological response modifiers, and other endocrine therapies, and long-term systemic corticosteroids, hormone replacement therapy, and prophylactic bisphosphonate drugs, were prohibited during the study.

\section{Study endpoints}

The primary endpoints of the present analysis were the occurrence of grade 3-4 adverse events, serious adverse events, changes in bone mineral density, and quality-of-life scores in the two study groups. Additional endpoints evaluated included all other adverse events and disease-free survival (DFS)-related events, including local recurrences, distant metastases, and secondary primary cancers or death.

All adverse events occurring during the study were recorded and graded in accordance with the National Cancer Institute Common Toxicity Criteria (version 3.0). However, only those adverse events considered related to the study medications are reported here.

Bone mineral density (BMD) of the lumbar spine was measured using dual-energy X-ray absorptiometry at the time of randomization, at 6 and 18 months of treatment, and at 6 months after the last treatment. The BMD assessments were blinded and centrally reviewed. T-scores were determined according to WHO criteria as SD units from the mean BMD of young, healthy women. The WHO-defined BMD classifications that were used to categorize patients were: normal $=\mathrm{T}$-score $\geq-1.0$; osteopenia $=\mathrm{T}$-score between -1.0 and -2.5 ; osteoporosis $=\mathrm{T}$-score $\leq-2.5) .{ }^{15}$

Quality of life in the two study groups was evaluated at $\geq 12$ months after randomization via the Functional
Assessment of Cancer Therapy-Breast (FACT-B) questionnaire (version 4) ${ }^{16}$ and the Brief Index of Sexual Functioning for Women (BISF-W) questionnaire. ${ }^{17}$ FACT-B (version 4) consists of the 27-item FACT-General and the 9-item Breast Cancer subscales. All FACT questionnaire forms use 5-grade (0-4) scales. The BISF-W questionnaire consists of eleven items, using 5-grade (1-5) scales. The higher the score, the better is the satisfaction.

\section{Statistical analysis}

The sample size/power calculation was based on testing the primary hypothesis: the 3-year DFS rates of the tamoxifen group and the ADD group were $70 \%$ and $79.6 \%$, respectively. This corresponded to the HR risk ratio with $80 \%$ power and a two-sided 5\% significance level. In order to detect a difference of $9.6 \%$ between the two groups, the corresponding risk ratio was 0.68 , the rate of loss of follow-up was $5 \%$, and 160 events occurred in a total of 670 patients (335 patients per group). Randomization was performed by filling in randomization forms and faxing them to the data processing center. The patients were randomly assigned via random numbers to the treatment group by the designated service provider using an Interactive Voice Response System.

Descriptive statistics were used to verify the comparability of the demographic and baseline characteristics of the two study groups. Safety data, including grade 3-4 adverse events and serious adverse events, changes in BMD, and quality-oflife scores were also analyzed with descriptive statistics. DFS rates during follow-up were estimated and compared using the Kaplan-Meier method. Continuous variables were tested if they presented normal distribution using the $F$-test. The results of the two groups were compared using the $t$-test or the Mann-Whitney $U$ test for parametric and nonparametric data, respectively. Chi-squared test was used for categorical variables. Qualitative variables were compared using the chisquared test with Yates correction or the Fisher's exact test, when necessary. Unless specifically indicated, all tests were two sided, and $P$-values $<0.05$ were considered statistically significant. Analyses were performed by using SAS software, version 9.2 (SAS Institute, Cary, NC, USA).

\section{Results}

From January 2008 to July 2011, 77 patients treated at eight centers in People's Republic of China were screened for the trial. Of these, 62 patients were randomized and found to be eligible for analysis (Table 1). The median age of the analyzed population was 41.0 years (range $9-51$ years); the ADD group comprised 33 patients and the TAM group 29 patients. The 
Table I Trial profile

\begin{tabular}{|l|l|l|l|}
\hline & $\begin{array}{l}\text { ADD } \\
\text { group }\end{array}$ & $\begin{array}{l}\text { TAM } \\
\text { group }\end{array}$ & Total \\
\hline No. assessed for eligibility & $I$ & $I$ & 77 \\
No. randomly allocated & 33 & 29 & 62 \\
No. discontinued treatment & $6(18.2 \%)$ & $6(20.7 \%)$ & $\mathrm{I} 2(19.4 \%)$ \\
Due to adverse events & $3(9.1 \%)$ & 0 & $3(4.8 \%)$ \\
Due to protocol violation & $2(6.1 \%)$ & $3(10.3 \%)$ & $5(8.1 \%)$ \\
Due to withdrawal of consent & $\mathrm{I}(3.0 \%)$ & $\mathrm{I}(3.4 \%)$ & $2(3.2 \%)$ \\
Due to patients lost to follow-up & 0 & $\mathrm{I}(3.4 \%)$ & $\mathrm{I}(\mathrm{I} .6 \%)$ \\
Due to management mistakes & 0 & $\mathrm{I}(3.4 \%)$ & $\mathrm{I}(\mathrm{I} .6 \%)$ \\
\hline
\end{tabular}

Note: ane patient found with brain metastasis after randomization.

Abbreviations: ADD, anastrozole + goserelin, TAM, tamoxifen.

ages, body mass index, hormone receptor status, disease stage, HER-2 status, and previous treatments of the patients in the two study groups are shown in Table 2 . There were no statistically significant differences between the two groups at baseline.

\section{Safety analysis}

A total of 13 patients (21\%) experienced 25 treatment-related adverse events during the study (Table 3), 11 of whom (33.3\%) were in the ADD group and $2(6.9 \%)$ in the TAM group. Arthralgia was the most common adverse event in the ADD group $(5 / 33 ; 15.2 \%)$ and two patients in this group had fractures. Three patients withdrew from the trial because of adverse events, all of whom were in the ADD group, while four patients required temporary suspension or a dosage reduction due to adverse events, three patients in the ADD group (9.1\%) and one patient in the TAM group (3.4\%). There were no instances of significant hematologic adverse events in either group.

An overall summary of adverse events in the two patient groups, including the numbers with grade 3 or 4 events is shown in Table 4.

\section{Changes in BMD}

At baseline, the mean lumbar spine BMD T-scores were $-0.56 \pm 0.31$ in ADD group and $-0.42 \pm 0.37$ in TAM group $(P=0.77)$. After $\geq 6$ months of treatment, there were no significant differences between the two groups $(P=0.54)$. The mean changes in T-scores after $\geq 6$ months of treatment were $-0.22 \pm 0.23$ in the ADD group $(P=0.69)$ and $-0.04 \pm 0.09$ in the TAM group $(P=0.89)$. Normal BMD, osteopenia, and osteoporosis were noted in $56.3 \%, 37.5 \%$, and $6.3 \%$ of patients, respectively, in the ADD group and 50.0\%, 50.0\%, and $0 \%$ of patients, respectively, in the TAM group.

\section{Quality-of-life scores}

Quality-of-life scores for 20 patients who completed the FACT-B and BISF-W questionnaires are shown in Table 5. No significant differences were evident between the two patient groups for any of the quality-of-life domains assessed, including physiological status, social and family circumstances, emotional state, functional status, and satisfaction with sex life $(P>0.05$ in all cases).

\section{DFS-related events}

After a median follow-up of 34 months (ADD group 34.4 months, TAM group 31.8 months), five patients in the ADD group (15.2\%) had recurrences or distant metastases as compared with three (10.3\%) in the TAM group. The sites of metastasis included bone, liver, supraclavicular lymph nodes, and the lung. In the TAM group, one patient developed a secondary primary malignancy (thyroid cancer).

At 24 months after randomization, the DFS rate was $\sim 80 \%$ in both groups ( $P=0.77$; Figure 1$)$. No deaths or contralateral secondary primary breast cancers occurred during the study.

\section{Discussion}

In SOFT and TEXT, the 5-year rates of recurrence of breast cancer were significantly lower among premenopausal women who received the AI exemestane plus ovarian suppression than among those who received tamoxifen plus ovarian suppression. The addition of ovarian suppression to tamoxifen did not result in significantly lower recurrence rates compared to those treated with tamoxifen alone. ${ }^{14}$ In 2018, they reported the updated results showing that in SOFT, the 8-year DFS rate was $78.9 \%$ with tamoxifen alone, $83.2 \%$ with tamoxifen plus ovarian suppression, and $85.9 \%$ with exemestane plus ovarian suppression $(P=0.009)$. Grade 3 or higher adverse events were reported in $24.6 \%$ of the tamoxifen-alone group, $31.0 \%$ of the tamoxifen-ovarian suppression group, and $32.3 \%$ of the exemestane-ovarian suppression group. ${ }^{18} \mathrm{We}$, therefore, suspended the enrollment of patients in our study after 62 patients had been randomized and followed up, and reported the summary of the safety, quality of life, and preliminary efficacy data of this population. Our findings showed that switching to AI plus GnRH-a therapy was associated with more frequent adverse events of all grades than continued tamoxifen therapy (33.3\% vs $6.9 \%$, respectively) and with more frequent grade 3 adverse events (15.2\% vs $0 \%)$. This finding is similar to the results of studies in postmenopausal patients who switched from tamoxifen to AI therapy. ${ }^{3,4}$ In the ADD group of the present study, the higher incidence of adverse events was mainly due to more joint and soft tissue pains, which has also been reported in previous studies of the concurrent use of GnRH-a ${ }^{19}$ and AI therapy, ${ }^{20}$ although 
Table 2 Patient demographics and clinical characteristics

\begin{tabular}{|c|c|c|c|}
\hline Characteristic & ADD group $(n=33)$ & TAM group $(n=29)$ & $P$-value \\
\hline \multicolumn{4}{|l|}{ Age, years } \\
\hline Median & 41 & 41 & 0.56 \\
\hline Range & $32-50$ & $29-51$ & \\
\hline \multicolumn{4}{|l|}{ Weight, kg } \\
\hline Median & 59 & 55 & 0.78 \\
\hline Range & $38-74$ & $42-77$ & \\
\hline \multicolumn{4}{|l|}{ Height, cm } \\
\hline Median & 160 & 160 & 0.90 \\
\hline Range & $146-172$ & $150-165$ & \\
\hline \multicolumn{4}{|l|}{ BMI, kg/m² } \\
\hline Median & 23.5 & 23.0 & 0.71 \\
\hline Range & $|8-3|$ & $16-29$ & \\
\hline Menopausal status ${ }^{\mathrm{a}}, \mathrm{n}(\%)$ & & & 0.15 \\
\hline I & $26(78.8 \%)$ & $18(62.1 \%)$ & \\
\hline 2 & 5 (I5.2\%) & $7(24.1 \%)$ & \\
\hline 3 & $2(6.1 \%)$ & $2(6.9 \%)$ & \\
\hline 4 & 0 & $2(6.9 \%)$ & \\
\hline Histology & & & l \\
\hline Invasive ductal carcinoma & $33(100 \%)$ & $29(100 \%)$ & \\
\hline Invasive lobular carcinoma & 0 & 0 & \\
\hline \multicolumn{4}{|l|}{ Tumor grade } \\
\hline Low & 0 & 0 & \\
\hline Intermediate & $20(60.6 \%)$ & $18(62.1 \%)$ & 0.75 \\
\hline High & $3(9.1 \%)$ & $2(6.9 \%)$ & \\
\hline Unknown & $10(30.3 \%)$ & 9 (31.0\%) & \\
\hline Estrogen receptor status, n (\%) & & & 0.31 \\
\hline Negative & $5(15.2 \%)$ & $2(6.9 \%)$ & \\
\hline Positive & $28(84.8 \%)$ & $27(93.1 \%)$ & \\
\hline Progesterone receptor status, $\mathrm{n}(\%)$ & & & 0.21 \\
\hline Negative & $4(12.1 \%)$ & I (3.4\%) & \\
\hline Positive & $29(87.9 \%)$ & $28(96.6 \%)$ & \\
\hline HER-2 status, n (\%) & & & 0.80 \\
\hline Negative & $26(78.8 \%)$ & $24(82.8 \%)$ & \\
\hline Positive & $4(12.1 \%)$ & $3(10.3 \%)$ & \\
\hline Unknown & $3(9.1 \%)$ & $2(6.9 \%)$ & \\
\hline Median duration of follow-up, months & 34.38 & 31.77 & 0.34 \\
\hline Range & $9.10-51.77$ & $17.03-58.57$ & \\
\hline IQR & $26.13-43.03$ & $25.00-40.79$ & \\
\hline Axillary nodes, $n$ (\%) & & & 0.49 \\
\hline pNo & $4(12.1 \%)$ & $2(7.1 \%)$ & \\
\hline $\mathrm{pNI}$ & $17(51.5 \%)$ & $21(75.0 \%)$ & \\
\hline $\mathrm{pN} 2$ & $9(27.3 \%)$ & $4(14.3 \%)$ & \\
\hline $\mathrm{pN} 3$ & $3(9.1 \%)$ & I (3.6\%) & \\
\hline Unknown & 0 & I (3.4\%) & \\
\hline Tumor size, $\mathrm{n}(\%)$ & & & 0.57 \\
\hline pTI & $9(27.3 \%)$ & $15(51.7 \%)$ & \\
\hline $\mathrm{pT} 2$ & $19(57.6 \%)$ & II (37.9\%) & \\
\hline PT3-4 & $2(6.1 \%)$ & I (3.4\%) & \\
\hline Unknown & $3(9.1 \%)$ & $2(6.9 \%)$ & \\
\hline Distant metastases & 0 & $\mathrm{I}^{\mathrm{b}}(3.4 \%)$ & l \\
\hline \multicolumn{4}{|l|}{ Previous treatment, n (\%) } \\
\hline \multicolumn{4}{|l|}{ Chemotherapeutic regimens } \\
\hline Taxane based & $9(27.3 \%)$ & $8(27.6 \%)$ & 0.97 \\
\hline Anthracycline based & $28(84.8 \%)$ & $27(93.1 \%)$ & 0.31 \\
\hline Neoadjuvant chemotherapy & $6(18.2 \%)$ & $6(20.7 \%)$ & 0.80 \\
\hline Radiotherapy & $18(54.5 \%)$ & $18(62.1 \%)$ & 0.55 \\
\hline
\end{tabular}

Notes: ${ }^{M}$ Menopausal status: I=normal menstruation; $2=$ amenorrhea of not more than 6 months' duration prior to enrollment and circulating blood levels of E2, FSH, and $\mathrm{LH}$ not at postmenopausal levels; $3=$ younger than 40 years with amenorrhea of not more than I year duration and circulating blood levels of E2, $\mathrm{FSH}$, and $\mathrm{LH}$ not at postmenopausal levels; $4=$ hysterectomy prior to enrollment with one or both ovaries retained and circulating blood levels of E2, FSH, and LH not at postmenopausal levels. bone patient found with brain metastasis after randomization.

Abbreviations: ADD, anastrozole + goserelin; BMI, body mass index; E2, estradiol; FSH, follicle-stimulating hormone; LH, luteinizing hormone, TAM; tamoxifen. 
Table 3 Drug-related adverse events (n, \%)

\begin{tabular}{|c|c|c|c|}
\hline & ADD group $(n=33)$ & TAM group $(n=29)$ & P-value \\
\hline No. of patients with adverse events & II (33.3\%) & $2(6.9 \%)$ & 0.01 \\
\hline Fractures & $2(6.0 \%)$ & 0 & 0.18 \\
\hline Arthralgia & $5(15.2 \%)$ & I (3.4\%) & 0.12 \\
\hline Myalgia & $2(6.1 \%)$ & 0 & 0.18 \\
\hline Ligament sprain & $\mathrm{I}(3.0 \%)$ & 0 & 0.355 \\
\hline Headache & $2(6.1 \%)$ & 0 & 0.18 \\
\hline Dizziness & I (3.0\%) & 0 & 0.35 \\
\hline Dyskinesia & $\mathrm{I}(3.0 \%)$ & 0 & 0.35 \\
\hline Hot flashes & $3(9.1 \%)$ & 0 & 0.10 \\
\hline ALT increased & I (3.0\%) & 0 & 0.35 \\
\hline Weight gain & $\mathrm{I}(3.0 \%)$ & 0 & 0.35 \\
\hline Night sweat & $\mathrm{I}(3.0 \%)$ & 0 & 0.35 \\
\hline Fatigue & 0 & $\mathrm{I}(3.4 \%)$ & 0.28 \\
\hline Eye pain & $2(6.1 \%)$ & 0 & 0.18 \\
\hline Decreased appetite & 0 & I (3.4\%) & 0.28 \\
\hline Severe depression & I (3.0\%) & 0 & 0.35 \\
\hline Eyelid edema & I (3.0\%) & 0 & 0.35 \\
\hline Alopecia & 0 & I (3.4\%) & 0.28 \\
\hline Abdominal discomfort & I (3.0\%) & 0 & 0.35 \\
\hline
\end{tabular}

Abbreviations: ADD, anastrozole + goserelin; ALT, alanine aminotransferase; TAM, tamoxifen.

Table 4 Overall summary of adverse events

\begin{tabular}{|l|l|l|l|}
\hline & ADD group (n=33) & TAM group (n=29) & P-value \\
\hline No. of patients with at least one drug-related adverse event, $n(\%)$ & $11(33.3 \%)$ & $2(6.9 \%)$ & 0.01 \\
No. with adverse events that led to treatment withdrawal, $\mathrm{n}(\%)$ & $3(9.1 \%)$ & 0 & 0.10 \\
No. with adverse events that led to temporary suspension of & $3(9.1 \%)$ & $\mathrm{I}(3.4 \%)$ & 0.37 \\
treatment or dosage reduction, $\mathrm{n}(\%)$ & 0 & 0 & - \\
No. of deaths & $5(15.2 \%)$ & 0 & 0.03 \\
No. with grade 3 adverse events & 0 & 0 & - \\
No. with grade 4 adverse events & & \\
\hline
\end{tabular}

Abbreviations: ADD, anastrozole + goserelin; TAM, tamoxifen.

Table 5 Quality-of-life scores for the FACT-B and BISF-W questionnaires at $\geq 12$ months after randomization

\begin{tabular}{|l|l|l|l|l|l|l|}
\hline Questionnaire/domain & $\begin{array}{l}\text { Treatment } \\
\text { group }\end{array}$ & $\mathbf{n}$ & $\begin{array}{l}\text { Mean } \\
\text { score }\end{array}$ & SD & $\begin{array}{l}\text { Standard error } \\
\text { (mean) }\end{array}$ & $\begin{array}{l}\text { P-value } \\
\text { (two-tailed test) }\end{array}$ \\
\hline FACT-B questionnaire & & & & & & \\
Physiological status & ADD & 10 & 20.30 & 4.423 & 1.399 & 0.96 \\
& TAM & 10 & 20.20 & 3.853 & 1.218 & 0.56 \\
Social and family circumstances & ADD & 10 & 20.79 & 7.828 & 2.476 & 0.27 \\
Emotional state & TAM & 10 & 22.37 & 3.319 & 1.049 & \\
& ADD & 10 & 18.30 & 3.529 & 1.116 & 0.56 \\
Functional status & TAM & 10 & 16.56 & 3.136 & 0.992 & \\
Additional concerns & ADD & 10 & 16.37 & 7.997 & 2.529 & \\
BISF-W questionnaire & TAM & 10 & 18.00 & 3.266 & 1.033 & \\
Sexual satisfaction & ADD & 10 & 19.10 & 4.999 & 1.581 & \\
& TAM & 10 & 20.70 & 5.813 & 1.838 & \\
& ADD & 9 & 33.11 & 8.223 & 2.741 & \\
\end{tabular}

Abbreviations: ADD, anastrozole + goserelin; BISF-W, Brief Index of Sexual Functioning for Women; FACT-B, Functional Assessment of Cancer Therapy-Breast; TAM, tamoxifen. 


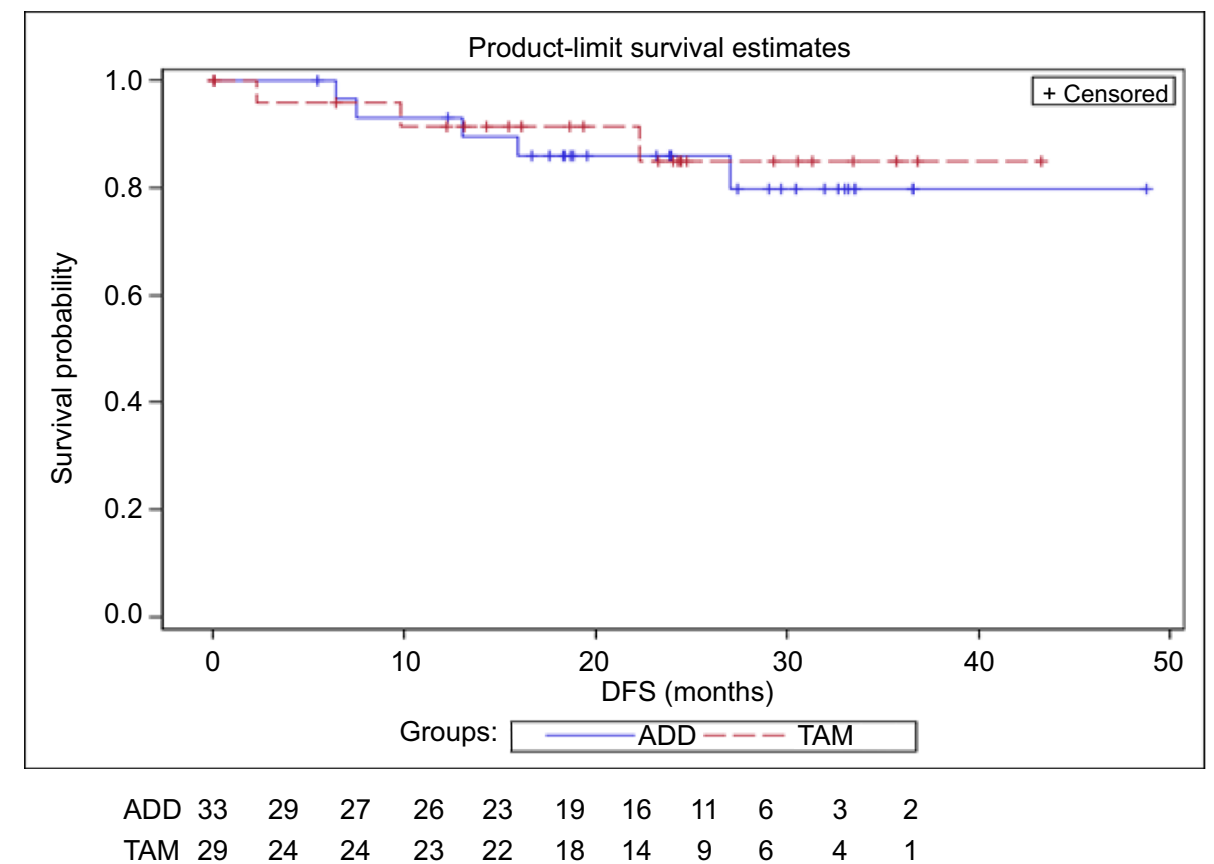

Figure I Kaplan-Meier analysis of DFS over 24 months after randomization.

Abbreviations: ADD, anastrozole + goserelin; DFS, disease-free survival; TAM, tamoxifen.

the specific mechanism of this adverse effect is still unclear. Menopausal-related symptoms, such as hot flashes, are common adverse events with adjuvant tamoxifen therapy of premenopausal breast cancer. GnRH-a, which causes significant reduction of estrogen levels in premenopausal patients, has been reported to produce more menopausal-related symptoms than tamoxifen alone in the study that compared buserelin, tamoxifen, and both agents combined in premenopausal breast cancer patients. ${ }^{21}$ However, in the present study, patients had been receiving tamoxifen treatment for $2-3$ years beforehand. Most of them may have already tolerated menopausal-related symptoms. So, no menopausal-related symptoms were reported in the TAM group and the incidence of hot flashes in the ADD group was $<10 \%$.

Osteoporosis and fractures are the major adverse events of adjuvant GnRH-a therapy of premenopausal breast cancer patients ${ }^{6}$ and of AI therapy in postmenopausal breast cancer patients. ${ }^{3,4}$ Therefore, it is possible that a combination of both therapies may produce overlapping adverse events in premenopausal patients, leading to a significant reduction in the BMD of lumbar vertebrae or the upper femur within 6 months of starting such treatment. ${ }^{11}$ However, the present study has shown that osteopenia was not aggravated in the ADD group in comparison with the TAM group, despite two fractures occurring in the ADD group. To date, the long-term effects of $\mathrm{AI}+\mathrm{GnRH}-\mathrm{a}$ combination therapy in comparison with tamoxifen alone on bone quality in early-stage premenopausal breast cancer patients have not been reported in the literature. Although tamoxifen definitely provides some protection against bone loss for postmenopausal patients, it also decreases BMD in patients who receive it prior to menopause. ${ }^{22}$ As all patents in the present study were premenopausal prior to enrollment, the results of our analysis showing that osteopenia was present in $50 \%$ of patients in the TAM group indicate that possible bone loss with long-term use of tamoxifen should not be neglected. Although preventive therapy with diphosphonates such as zoledronic acid was not employed in the present study, this kind of therapy has been demonstrated to prevent a decrease in BMD caused by $\mathrm{AI}+\mathrm{GnRH}-\mathrm{a}$ treatment in other studies. ${ }^{23,24}$

\section{Conclusion}

This trial explores the feasibility of switching premenopausal patients from tamoxifen to GnRH-a+AIs after 2-3 years of tamoxifen therapy. Unfortunately, our results did not provide evidence that the switch can improve the efficacy. While an increased occurrence of adverse effects was observed in the GnRH-a+AIs treatment group (especially, bone-related events), treatment was still well tolerated and did not affect the patients' quality of life. While future study may be required for further evidence, for patients who cannot tolerate the side effects of tamoxifen or have tamoxifen 
contraindications, GnRH-a plus AIs plus diphosphonate treatment may be a viable alternative treatment.

\section{Data sharing statement}

The authors will share individual de-identified participant data as any specific data or any other study-related documents by any accessible way at any time as long as possible.

\section{Acknowledgments}

Funding for this study was provided by the Chinese Breast Cancer Society Group (CBCSG). Editorial assistance was provided by ContentEdNet, Shanghai Co. Ltd. The abstract of this paper was presented at 18th ECCO - 40th ESMO European Cancer Congress which was held in Vienna, Austria from 25 to 29 September 2015 as a poster presentation with interim findings. The poster's abstract was published in "Poster Abstracts" in European Journal of Cancer: Hyperlink with DOI: 10.1016/S0959-8049(16)30896-6.

\section{Disclosure}

The authors report no conflicts of interest in this work.

\section{References}

1. Sabnis GJ, Macedo LF, Goloubeva O, Schayowitz A, Brodie AM. Stopping treatment can reverse acquired resistance to letrozole. Cancer Res. 2008;68(12):4518-4524.

2. Tang LC, Yin WJ, Di GH, Shen ZZ, Shao ZM. Unfavourable clinicopathologic features and low response rate to systemic adjuvant therapy: results with regard to poor survival in young Chinese breast cancer patients. Breast Cancer Res Treat. 2010;122(1):95-104.

3. Coombes RC, Hall E, Gibson LJ, et al. A randomized trial of exemestane after two to three years of tamoxifen therapy in postmenopausal women with primary breast cancer. $N$ Engl J Med. 2004;350(11):1081-1092.

4. Jakesz R, Jonat W, Gnant M, et al. Switching of postmenopausal women with endocrine-responsive early breast cancer to anastrozole after 2 years' adjuvant tamoxifen: combined results of ABCSG trial 8 and ARNO 95 trial. Lancet. 2005;366(9484):455-462.

5. Tamoxifen for early breast cancer: an overview of the randomised trials. Early Breast Cancer Trialists' Collaborative Group. Lancet. 1998;351(9114):1451-1467.

6. Baum M, Hackshaw A, Houghton J, et al. Adjuvant goserelin in premenopausal patients with early breast cancer: results from the ZIPP study. Eur J Cancer. 2006;42(7):895-904.

7. Castiglione-Gertsch M, O’Neill A, Price KN, et al. Adjuvant chemotherapy followed by goserelin versus either modality alone for premenopausal lymph node-negative breast cancer: a randomized trial. J Natl Cancer Inst. 2003;95:1833-1846.

8. Karlsson P, Sun Z, Braun D, et al. Long-term results of International Breast Cancer Study Group Trial VIII: adjuvant chemotherapy plus goserelin compared with either therapy alone for premenopausal patients with node-negative breast cancer. Ann Oncol. 2011;22(10):2216-2226.
9. Carlson RW, Theriault R, Schurman CM, et al. Phase II trial of anastrozole plus goserelin in the treatment of hormone receptor-positive, metastatic carcinoma of the breast in premenopausal women. J Clin Oncol. 2010;28(25):3917-3921.

10. Torrisi R, Bagnardi V, Rotmensz N, et al. Letrozole plus GnRH analogue as preoperative and adjuvant therapy in premenopausal women with ER positive locally advanced breast cancer. Breast Cancer Res Treat. 2011;126(2):431-441.

11. Masuda N, Sagara Y, Kinoshita T, et al. Neoadjuvant anastrozole versus tamoxifen in patients receiving goserelin for premenopausal breast cancer (STAGE): a double-blind, randomised Phase 3 trial. Lancet Oncol. 2012;13(4):345-352.

12. Jannuzzo MG, di Salle E, Spinelli R, Pirotta N, Buchan P, Bello A. Estrogen suppression in premenopausal women following 8 weeks of treatment with exemestane and triptorelin versus triptorelin alone. Breast Cancer Res Treat. 2009;113(3):491-499.

13. Gnant M, Mlineritsch B, Stoeger H, et al. Adjuvant endocrine therapy plus zoledronic acid in premenopausal women with early-stage breast cancer: 62-month follow-up from the ABCSG-12 randomised trial. Lancet Oncol. 2011;12(7):631-641.

14. Pagani O, Regan MM, Walley BA, et al. Adjuvant exemestane with ovarian suppression in premenopausal breast cancer. $N$ Engl J Med. 2014;371(2):107-118.

15. Prevention and management of osteoporosis. World Health Organ Tech Rep Ser. 2003;921:1-164

16. Brady MJ, Cella DF, Mo F, et al. Reliability and validity of the functional assessment of cancer therapy-breast quality-of-life instrument. $J$ Clin Oncol. 1997;15(3):974-986.

17. Mazer NA, Leiblum SR, Rosen RC. The brief index of sexual functioning for women (BISF-W): a new scoring algorithm and comparison of normative and surgically menopausal populations. Menopause. 2000;7(5):350-363.

18. Francis PA, Pagani O, Fleming GF, et al. Tailoring adjuvant endocrine therapy for premenopausal breast cancer. N Engl J Med. 2018;379(2): 122-137.

19. Bernhard J, Zahrieh D, Castiglione-Gertsch M, et al. Adjuvant chemotherapy followed by goserelin compared with either modality alone: the impact on amenorrhea, hot flashes, and quality of life in premenopausal patients - the International Breast Cancer Study Group Trial VIII. J Clin Oncol. 2007;25(3):263-270.

20. Forbes JF, Cuzick J, Buzdar A, et al. Effect of anastrozole and tamoxifen as adjuvant treatment for early-stage breast cancer: 100-month analysis of the ATAC trial. Lancet Oncol. 2008;9:45-53.

21. Klijn JG, Beex LV, Mauriac L, et al. Combined treatment with buserelin and tamoxifen in premenopausal metastatic breast cancer: a randomized study. J Natl Cancer Inst. 2000;92(11):903-911.

22. Powles TJ, Hickish T, Kanis JA, Tidy A, Ashley S. Effect of tamoxifen on bone mineral density measured by dual-energy X-ray absorptiometry in healthy premenopausal and postmenopausal women. J Clin Oncol. 1996;14(1):78-84.

23. Gnant M, Mlineritsch B, Luschin-Ebengreuth G, et al. Adjuvant endocrine therapy plus zoledronic acid in premenopausal women with earlystage breast cancer: 5-year follow-up of the ABCSG-12 bone-mineral density substudy. Lancet Oncol. 2008;9(9):840-849.

24. Gnant MF, Mlineritsch B, Luschin-Ebengreuth G, et al. Zoledronic acid prevents cancer treatment-induced bone loss in premenopausal women receiving adjuvant endocrine therapy for hormone-responsive breast cancer: a report from the Austrian Breast and Colorectal Cancer Study Group. J Clin Oncol. 2007;25(7):820-828. 
Cancer Management and Research

\section{Publish your work in this journal}

Cancer Management and Research is an international, peer-reviewed open access journal focusing on cancer research and the optimal use of preventative and integrated treatment interventions to achieve improved outcomes, enhanced survival and quality of life for the cancer patient.

The manuscript management system is completely online and includes

Submit your manuscript here: https://www.dovepress.com/cancer-management-and-research-journal

a very quick and fair peer-review system, which is all easy to use. Visit $\mathrm{http}: / / \mathrm{www}$.dovepress.com/testimonials.php to read real quotes from published authors. 